\title{
Long-term outcomes and prognostic factor analysis of resected Siewert type II adenocarcinoma of esophagogastric junction in China: a seven-year study
}

\author{
Yiding Feng ${ }^{1}$, Youhua Jiang ${ }^{1}$, Qiang Zhao ${ }^{1}$, Jinshi Liu' ${ }^{1}$, Hangyu Zhang ${ }^{2}$ and Qixun Chen ${ }^{1 *}$ (D)
}

\begin{abstract}
Background: The incidence rate of adenocarcinoma of the esophagogastric junction (AEG) has significantly increased over the past two decades. Surgery remains the only curative treatment. However, there are currently few studies on Chinese AEG patients. The purpose of this study was to retrospectively analyze the survival and prognostic factors of AEG patients in our center.

Methods: Between January 2008 and September 2014, 249 AEG patients who underwent radical resection were enrolled in this retrospective study, including 196 males and 53 females, with a median age of 64 (range 31-82). Prognostic factors were assessed with the log-rank test and Cox univariate and multivariate analyses.

Results: The 5-year survival rate of all patients was 49\%. The median survival time of all enrolled patients was 70.1 months. Pathological type, intraoperative blood transfusion, tumor size, adjuvant chemotherapy, duration of hospital stay, serum CA199, CA125, CA242 and CEA, pTNM stage, lymphovascular or perineural invasion, and the ratio of positive to negative lymph nodes (PNLNR) were significantly associated with overall survival when analyzed in univariate analysis.

Conclusions: Our study found that adjuvant chemotherapy, PNLNR, intraoperative blood transfusion, tumor size, perineural invasion, serum CEA, and duration of hospital stay after surgery had significance in multivariate analysis and were independent risk factors for survival.
\end{abstract}

Keywords: Esophagogastric junction adenocarcinoma, Lymph nodes, Siewert classification, Surgery

\section{Background}

Adenocarcinoma of the esophagogastric junction (AEG) has been reported to account for approximately $5-8 \%[1$, 2] of all esophageal cancers in China and $35.7 \%$ of gastric cancers and lower esophageal cancers worldwide [3]. Many population-based studies have shown that the

\footnotetext{
*Correspondence: chenqixun64@sina.com

1 Department of Thoracic Surgery, Cancer Hospital of the University

of Chinese Academy of Sciences(Zhejiang Cancer Hospital),

Hangzhou 31000, China

Full list of author information is available at the end of the article
}

incidence rate of AEG has significantly increased over the past two decades, both in Western countries and in East Asia [4-6]. The reported seven-fold increase in the morbidity rate of AEG [7], which is a more substantial increase compared to that other malignancies, has led to a steady increase in the mortality rate from $2-15 / 100,000$ patients [8].

Surgical resection is the main curative treatment for AEG. Unlike the treatment for gastric cancer, which is standard surgical resection plus D2 lymph node resection, surgery for AEG is still controversial in many ways, 
especially for Siewert type II AEG. The surgical treatment of AEG includes primary tumor removal, lymph node dissection and reconstruction of the digestive tract. Regional lymph node metastasis is the most common metastasis method of AEG. Studies have found that the lymph node metastasis rate of AEG is $76.3 \%$, much higher than that of distal gastric adenocarcinoma (67.4\%) [9]. Moreover, the distribution of lymph node metastasis in different Siewert subtypes was different, resulting in several different surgical approaches $[10,11]$, and there are still controversies regarding the $\mathrm{N}$ staging of the AJCC TNM staging system [12]. Other controversial issues include the choice between laparoscopic surgery or open surgery and whether patients with AEG who achieve R0 resection should undergo neoadjuvant chemoradiotherapy.

Based on the above problems, although Siewert classified AEG in 2000, there are still many problems that exist in clinical practice. In China, squamous cell carcinoma remains the predominant pathological type of esophageal cancer. Therefore, esophageal adenocarcinoma has rarely been investigated among Chinese patients. Our study aimed to explore the long-term outcomes of AEG Chinese patients who underwent resection and to analyze the related prognostic factors.

\section{Methods}

\section{Patients}

From January 2008 to September 2014, there were 420 cases in which curative R0 resection was performed for esophagogastric junction cancers. Data from these 420 patients were collected, and the inclusion criteria were as follows: (I) patients with pathologically and immunohistochemically diagnosed AEG; (II) patients who underwent radical resection and did not have distant metastasis; and (III) patients with Siewert type II AEG. The exclusion criterion was patients without complete data for analysis. Among all collected patients, 249 patients had complete clinical data and confirmed postoperative pathology for adenocarcinoma and met all the inclusion and exclusion criteria.

Data on the demographics, comorbidities, pathologic details, surgical approach, blood infusion, duration of hospital stay, adjuvant therapy and survival time were collected and subsequently analyzed. Among the characteristics, the duration of hospital stay was classified according to whether the patient was hospitalized for longer than 10 days. The tumor size was classified according to whether the tumor size was larger than $4 \mathrm{~cm}$; the tumor pathology was subdivided according to whether there was lymphovascular invasion or perineural invasion. Because the number of lymph nodes dissected varied greatly among different patients, we specifically defined the ratio of positive to negative lymph nodes for all dissected lymph nodes to further differentiate the prognostic ability of different values, defined as the PNLNR (positive lymph nodes/negative lymph nodes ratio).

All postoperative patients in our center were followed up regularly. In general, the frequency of followup was every 3 months for the first 2 years, then every 6 months for the following 3 years, and annually thereafter. Telephone follow-up interviews were conducted at irregular intervals. This retrospective study was performed in accordance with the ethical standards of the Ethics Committee of Zhejiang Cancer Hospital and received Institutional Review Board approval. No informed consent was needed for this study.

\section{Surgical approach selection}

A reasonable surgical route should consider tumor resection, lymph node dissection, surgical margin and safety. The alternative AEG surgical approaches include left thoracotomy and the Ivor-Lewis, McKeown, and transhiatal or abdominal-transhiatal approaches. The selection of surgical approach referred to the NCCN guidelines for esophageal and esophagogastric junction cancers, NCCN guidelines for gastric cancer and Chinese expert consensus on the surgical treatment for adenocarcinoma of the esophagogastric junction. Preoperative staging was performed according to the eighth edition of the TNM staging system, and preoperative classification was performed based on the Siewert type.

In general, the abdominal-transhiatal approach was preferred for those with $<3 \mathrm{~cm}$ of esophagus involved, and the thoracotomy approach was preferred for those with $\geq 3 \mathrm{~cm}$ of esophagus involved.

\section{Statistical analyses}

All statistical calculations were performed with IBM SPSS Statistics (Version 19.0; IBM Corp., New York, USA). Charts were made with GraphPad Prism 6 (GraphPad Software, Inc., La Jolla, CA, USA). Overall survival (OS) was calculated from the date of surgery to the date of death due to any cause. The data of patients lost to follow-up were censored at the date of the last observation. The Cox proportional hazards model was used to determine the univariate and multivariate hazards ratios for the study parameters. Pearson product-moment correlation analysis was used to measure the relationship between two variables. For all tests, $\mathrm{P}<0.05$ was defined as statistically significant. 


\section{Results}

\section{Characteristics of the patients}

The baseline characteristics of the patients are shown in Tables 1, 2 and 3. There were 196 (78.7\%) males and 53 (21.3\%) females in the population. The median age of all enrolled patients was 64 years, ranging from $31-82$ years. The details of the pathological outcomes showed that most patients (72.3\%) had pure adenocarcinoma, and the remaining patients had mixed adenocarcinoma with signet ring or mucinous tumors. There were 165 (66.3\%) patients with tumor diameters larger than $4 \mathrm{~cm}$ and 84 (33.7\%) patients with tumor diameters less than or equal to $4 \mathrm{~cm}$. Among them, 127 (51.0\%) patients had lymphovascular invasion, and 154 (61.8\%) patients had perineural invasion. Pathological differentiation showed that only 2 patients were classified as G1, 92 (36.9\%) were classified as G2, and 155 (62.2\%) were classified as G3.

Among the patients who underwent lymph node dissection, 70 (28.1\%) patients had no positive lymph nodes, $32(12.9 \%)$ patients had a PNLNR greater than 1, and 147 (59.0\%) had a PNLNR less than 1. According to TNM staging, $23(9.2 \%)$ patients were defined as stage I, 45 (18.1\%) as stage II, 94 (37.8\%) as stage III and 87 (34.9\%) as stage IV.

In terms of surgery, there were a total of 3 types of surgical approaches, including $73(29.3 \%)$ cases of left thoracotomy, $1(0.4 \%)$ case of Ivor-Lewis surgery, and 175 (70.3\%) cases of transhiatal surgery. Because only one patient underwent Ivor-Lewis surgery, we excluded this patient in the univariate and multivariate analyses. Total gastrectomy was performed in 115 (46.2\%) patients, and proximal gastrectomy was performed in 134 (53.8\%) patients. During surgery, a small portion of patients $(\mathrm{n}=18)$ received intraoperative blood transfusions. After surgery, $53.4 \%$ patients stayed in the hospital for longer than 10 days, and $46.6 \%$ patients stayed for less than or equal to 10 days. Long hospital stays were mainly due to postoperative complications, postoperative nutritional status and several other reasons. Regarding adjuvant chemotherapy, $100(40.2 \%)$ patients received adjuvant chemotherapy, and the main chemotherapy regimens

Table 1 Clinical characteristics of 249 patients

\begin{tabular}{ll}
\hline Characteristics & Case No. (\%) \\
\hline Gender & \\
Male & $196(78.7)$ \\
Female & $53(21.3)$ \\
Age & \\
Range & $31-82$ \\
Median & 64 \\
\hline
\end{tabular}

Table 2 Surgery characteristics of 249 patients

\begin{tabular}{ll}
\hline Characteristics & Case no. (\%) \\
\hline Type of gastric resection & \\
Total gastrectomy & $115(46.2)$ \\
Proximal gastrectomy & $134(53.8)$ \\
Surgical approach & \\
Left thoracotomy & $73(29.3)$ \\
Ivor Lewis & $1(0.4)$ \\
Transhiatal & $175(70.3)$ \\
Intraoperative blood transfusion & \\
Yes & $231(92.8)$ \\
No & $18(7.2)$ \\
\hline
\end{tabular}

Table 3 Pathology and postoperative characteristics of 249 patients

\begin{tabular}{|c|c|}
\hline Characteristics & Case no. (\%) \\
\hline \multicolumn{2}{|l|}{ Type of pathology } \\
\hline Adenocarcinoma & $180(72.3)$ \\
\hline Adenocarcinoma with partial signet ring or mucinous & $69(27.7)$ \\
\hline \multicolumn{2}{|l|}{ Tumor size } \\
\hline$>4 \mathrm{~cm}$ & $165(66.3)$ \\
\hline$\leq 4 \mathrm{~cm}$ & $84(33.7)$ \\
\hline \multicolumn{2}{|l|}{ N stage } \\
\hline No & $71(28.5)$ \\
\hline N1 & $42(16.9)$ \\
\hline N2 & 49(19.7) \\
\hline N3 & $87(34.9)$ \\
\hline \multicolumn{2}{|l|}{ pTNM stage } \\
\hline 1 & $23(9.2)$ \\
\hline$\|$ & $45(18.1)$ \\
\hline III & $94(37.8)$ \\
\hline IV & $87(34.9)$ \\
\hline \multicolumn{2}{|l|}{ Differentiation } \\
\hline G1 & $2(0.8)$ \\
\hline G2 & $92(36.9)$ \\
\hline G3 & $155(62.2)$ \\
\hline \multicolumn{2}{|l|}{ Lymphovascular invasion } \\
\hline Yes & $127(51)$ \\
\hline No & $122(49)$ \\
\hline \multicolumn{2}{|l|}{ Perineuronal invasion } \\
\hline Yes & $154(61.8)$ \\
\hline No & $95(38.2)$ \\
\hline \multicolumn{2}{|l|}{ Adjuvant chemotherapy } \\
\hline Yes & $100(40.2)$ \\
\hline No & $149(59.8)$ \\
\hline \multicolumn{2}{|l|}{ Number of metastatic lymph nodes } \\
\hline Median (range) & $3(0-38)$ \\
\hline \multicolumn{2}{|l|}{ PNLNR } \\
\hline$>1$ & $32(12.9)$ \\
\hline$=0$ & $70(28.1)$ \\
\hline$\leq 1$ & $147(59)$ \\
\hline \multicolumn{2}{|l|}{ Postoperative hospital stays } \\
\hline$>10 d$ & $133(53.4)$ \\
\hline$\leq 10 \mathrm{~d}$ & $116(46.6)$ \\
\hline
\end{tabular}

PNLNR positive lymph nodes/ negative lymph nodes rate 
were SOX (S-1 and oxaliplatin) and XELOX (capecitabine and oxaliplatin).

\section{Survival data and prognostic factors}

We conducted our last follow-up in October 2019 by telephone interview or outpatient or inpatient department visits. The median follow-up time was approximately 75 months. At the last follow-up, 111 (44.6\%) patients were still alive. The 1-year, 3-year and 5-year survival rates of all enrolled patients were $72 \%, 59 \%$, and $49 \%$, respectively. The median survival time (mOS) of these patients was $70.1 \mathrm{~m}$ (95\% CI 53.6-86.6 m). Female patients had a significantly longer survival time than male patients (NA vs. $62.4 \mathrm{~m}, \mathrm{P}=0.039$ ). Patients with pure adenocarcinoma had a significantly longer survival time than mixed pathology patients (85.4 vs. $42.5 \mathrm{~m}, \mathrm{P}=0.011$ ). Patients with perineural invasion (NA vs. 48.1, $\mathrm{P}<0.001$ ) or lymphovascular invasion (NA vs. 40.9, $\mathrm{P}<0.001)$ had shorter survival times, received fewer blood transfusions $(12.4$ vs. $81.8, \mathrm{P}<0.001)$ had a shorter survival time (Fig. 1). Patients who received adjuvant chemotherapy (61.7 vs. $93.4, \mathrm{P}=0.027$ ) had a longer survival time. The median survival time of patients without lymph node metastasis was not reached, while the median survival time of N2 and N3 patients was $64.5 \mathrm{~m}$ and $24.0 \mathrm{~m}$, respectively. Regarding the PNLNR, the mOS of patients with a PNLNR $\leq 1$ was $67.2 \mathrm{~m}$ and that of patients with a PNLNR $>1$ was $11.8 \mathrm{~m}$ (Fig. 2). The mOS of patients with a tumor size greater than $4 \mathrm{~cm}$ was also not reached, while the mOS of those with a tumor size less than or equal to $4 \mathrm{~cm}$ was $43.8 \mathrm{~m}$ (Fig. 3). pTNM stage was also significantly related to survival time. Patients with elevated CA199, CEA, CA242 and AFP levels had shorter survival times (Table 4). Only 1 of 249 patients died within 30 days after surgery.

The prognostic factors included age, sex, pathological type, tumor size, type of surgical approach, serum

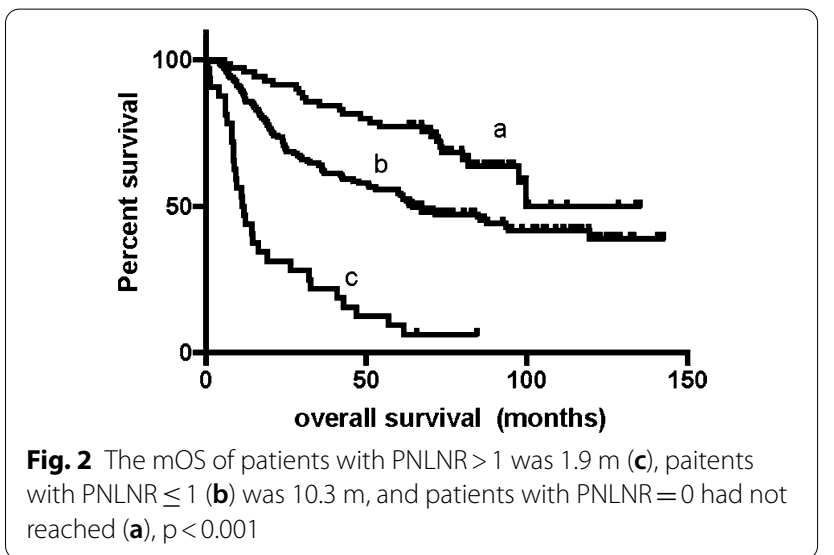

tumor biomarkers, and blood transfusion. Both univariate and multivariate analyses were applied (Tables 4 and 5). Among the factors related to survival in univariate analyses, 16 factors had significance, including sex, pathological type, intraoperative blood transfusion, surgical approach, and several serum tumor biomarkers. Factors including type of gastric resection, tumor differentiation, elevated serum AFP and serum ferritin had no significance. Then, multivariate analysis was conducted to identify the predictive indicators for a good prognosis using the parameters with a $\mathrm{P}$ value less than 0.5 in univariate analysis. Multivariate analysis showed that intraoperative blood transfusion, tumor size larger than $4 \mathrm{~cm}$, no adjuvant chemotherapy, higher positive/negative lymph node ratio, perineural invasion, elevated serum CEA before surgery, and length of stay in the hospital greater than 10 days after surgery were independent risk factors for survival in resected Siewert type II AEG patients.

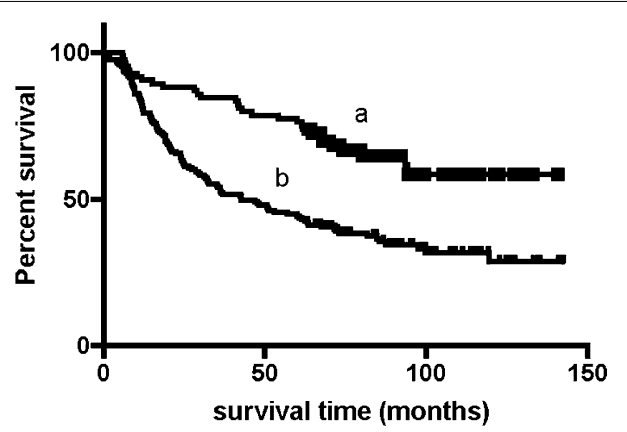

Fig. 3 The median overall survival (mOS) of patients with tumor diameter more than $4 \mathrm{~cm}$ had not reached (a), and mOS of tumor diameter less or equal to $4 \mathrm{~cm}$ was $43.8 \mathrm{~m} \mathrm{(b)}, \mathrm{p}<0.001$
Fig. 1 The mOS of patients without intraoperative blood transfusion is $81.8 \mathrm{~m} \mathrm{(a)}$ and patients with intraoperative blood transfusion is $12.4 \mathrm{~m}(\mathbf{b}), p<0.001$ 
Table 4 Univariate analysis of survival after surgery

\begin{tabular}{|c|c|c|c|c|}
\hline \multirow[t]{2}{*}{ Variables } & \multirow[t]{2}{*}{ MST (month) } & \multicolumn{2}{|c|}{ Univariate analysis } & \multirow[t]{2}{*}{ P-value } \\
\hline & & HR & $95 \% \mathrm{Cl}$ & \\
\hline Gender (male vs. female) & 62.4 vs. NA & 1.608 & $1.019-2.539$ & 0.039 \\
\hline Pathology (adeno vs. mix) & 85.4 vs. 42.5 & 0.632 & $0.442-0.903$ & 0.011 \\
\hline Blood transfusion (no vs. yes) & 12.4 vs. 81.8 & 0.262 & $0.156-0.440$ & $<0.001$ \\
\hline Surgical approaches (left thoracotomy vs. transhiatal) & 42.5 vs. 87.6 & 1.569 & $1.110-2.218$ & $<0.001$ \\
\hline Tumor size ( $\leq 4 \mathrm{~cm}$ vs. $>4 \mathrm{~cm})$ & NA vs. 43.8 & 0.41 & $0.274-0.616$ & $<0.001$ \\
\hline Adjuvant chemotherapy (no vs. yes) & 61.7 vs. 93.4 & 1.477 & $1.042-2.094$ & 0.027 \\
\hline Postoperative hospital stays ( $\leq 10 \mathrm{~d}$ vs. $>10 \mathrm{~d}$ ) & 59.9 vs. 99.7 & 0.806 & $0.680-0.956$ & 0.012 \\
\hline Type of gastric resection (total vs. proximal) & 73.5 vs. 64.5 & 0.96 & $0.811-1.135$ & 0.63 \\
\hline \multicolumn{5}{|l|}{ PNLNR } \\
\hline$=0$ & NA & 0.403 & $0.298-0.543$ & \\
\hline$\leq 1$ & 67.2 & 0.772 & $0.613-0.972$ & \\
\hline$>1$ & 11.8 & 1 & 1 & \\
\hline Perineuronal invasion (no vs. yes) & NA vs. 48.1 & 0.454 & $0.310-0.664$ & $<0.001$ \\
\hline Lymphovascular invasion (no vs. yes) & NA vs. 40.9 & 0.448 & $0.317-0.634$ & $<0.001$ \\
\hline Differentiation & 80 vs. 73 vs. 63 & & & 0.851 \\
\hline pTNM stage & & & & $<0.001$ \\
\hline । & 97.4 & 0.253 & $0.121-0.526$ & \\
\hline$\|$ & 99.7 & 0.265 & $0.155-0.451$ & \\
\hline III & 119.56 & 0.364 & $0.248-0.533$ & \\
\hline IV & 24.5 & 1 & 1 & \\
\hline Serum CA199 (U/ml) (<37 vs. $\geq 37)$ & 93.4 vs. 31.7 & 0.784 & $0.654-0.939$ & 0.008 \\
\hline Serum CEA (ng/ml) $(<5$ vs. $\geq 5)$ & 80.0 vs. 26.3 & 0.74 & $0.623-0.878$ & $<0.001$ \\
\hline Serum CA242 (U/ml) (<20 vs. $\geq 20)$ & 73.0 vs. 12.0 & 0.746 & $0.596-0.934$ & 0.01 \\
\hline Serum CA125 (U/ml) (<35 vs. $\geq 35)$ & 71.4 vs. 70.1 & 0.605 & $0.423-0.866$ & 0.004 \\
\hline $\operatorname{Serum} \operatorname{AFP}(\mathrm{ng} / \mathrm{ml})(<10 \mathrm{vs} . \geq 10)$ & 80.3 vs. 51.1 & 1.067 & $0.761-1.496$ & 0.706 \\
\hline Serum CA724 (U/ml) (<6.9vs. $\geq 6.9)$ & 70.1 vs. 80.0 & 0.862 & $0.715-1.039$ & 0.117 \\
\hline Serum ferritin (ng/ml) $(<274.66$ vs. $\geq 274.66)$ & & 0.952 & $0.708-1.280$ & 0.745 \\
\hline
\end{tabular}

\section{Discussion}

A number of various surgical approaches are available for Siewert type II AEG patients. With the increasing use of laparoscopic and thoracoscopic surgery, the surgical options have become more diversified. Various surgical approaches may lead to different numbers of dissected lymph nodes, and the surgical approach is strongly associated with prognosis.

Previous studies have suggested that the incidence of respiratory-related complications was noted to be higher in the thoracotomy group than in the transhiatal group [13-15]. In this study, only 1 patient underwent Ivor-Lewis surgery. There was no significant difference in postoperative length of hospital stay between the left thoracotomy group and the transhiatal group, with a median postoperative length of hospital stay of 11 days for both groups. However, survival was longer in the transhiatal group than in the thoracotomy group, which was consistent with previous similar studies [13]. Although no convincing theory so far can explain the difference between left thoracotomy and transhiatal thoracotomy, left thoracotomy is considered to be more invasive and has fewer survival benefits. This may be due to the much higher morbidity rate after thoracotomy, and an increasing number of studies have concluded that the transhiatal approach would be better for Siewert type 2 AEG patients.

In previous retrospective studies, perioperative blood transfusions were associated with poor prognosis after surgery for cancer and were a major independent risk factor for postoperative bacterial infection [16, 17]. Patients receiving intraoperative blood transfusion often have anemia before surgery or major intraoperative blood loss during surgery. This study found that the prognosis of patients who received intraoperative blood transfusions was poor. In this respect, laparoscopic surgery may be more advantageous as a minimally invasive surgical procedure.

When the number of positive lymph node metastases was included in the univariate analysis, it was concluded 
Table 5 Multiple cox regression analysis of survival after surgery

\begin{tabular}{|c|c|c|c|}
\hline \multirow[t]{2}{*}{ Variables } & \multicolumn{2}{|c|}{ Multivariate analysis } & \multirow[t]{2}{*}{ P-value } \\
\hline & HR & $95 \% \mathrm{Cl}$ & \\
\hline Gender (male vs. female) & 1.25 & $0.981-1.593$ & 0.071 \\
\hline Pathology (adeno vs. mix) & 1.066 & $0.864-1.314$ & 0.55 \\
\hline Blood transfusion (no vs. yes) & 0.63 & $0.471-0.843$ & 0.002 \\
\hline Surgical approaches (left thoracotomy vs. transhiatal) & 1.365 & $0.935-1.995$ & 0.107 \\
\hline Tumor size ( $\leq 4 \mathrm{~cm}$ vs. $>4 \mathrm{~cm})$ & 0.728 & $0.582-0.912$ & 0.006 \\
\hline Postoperative hospital stays ( $\leq 10 \mathrm{~d}$ vs. $>10 \mathrm{~d}$ ) & 0.833 & $0.694-0.999$ & 0.048 \\
\hline $\operatorname{PNLNR}(>1)$ & 1 & 1 & $<0.001$ \\
\hline PNLNR $(=0)$ & 0.561 & $0.268-1.175$ & 0.126 \\
\hline $\operatorname{PNLNR}(\leq 1)$ & 0.773 & $0.507-1.180$ & 0.233 \\
\hline Perineuronal invasion (no vs. yes) & 0.727 & $0.574-0.922$ & 0.008 \\
\hline Lymphovascular invasion (no vs. yes) & 0.899 & $0.721-1.121$ & 0.346 \\
\hline pTNM stage IV & 1 & 1 & 0.171 \\
\hline Stage III & 1.166 & $0.541-2.512$ & 0.694 \\
\hline Stage II & 0.868 & $0.442-1.707$ & 0.682 \\
\hline Stage I & 0.764 & $0.426-1.369$ & 0.366 \\
\hline Serum CA199 (U/ml) (<37 vs. $\geq 37)$ & 0.986 & $0.784-1.239$ & 0.902 \\
\hline Serum CEA (ng/ml) (<5 vs. $\geq 5)$ & 0.768 & $0.623-0.947$ & 0.014 \\
\hline Serum CA242 (U/ml) (<20 vs. $\geq 20)$ & 0.934 & $0.707-1.233$ & 0.629 \\
\hline Serum CA125 (U/ml) (<35 vs. $\geq 35)$ & 0.739 & $0.490-1.114$ & 0.148 \\
\hline Adjuvant chemotherapy (no vs. yes) & 1.478 & $1.214-1.800$ & $<0.001$ \\
\hline
\end{tabular}

that patients with different lymph node stages had significant survival differences, but in the multivariate analysis, the number of lymph node metastases was not an independent risk factor for this conclusion. However, the ratio of positive lymph nodes to negative lymph nodes (PNLNR) was significant in both univariate and multivariate analyses. This may be because the number of lymph nodes dissected in different surgeries varies greatly, and mere comparisons of the number of positive lymph nodes cannot accurately assess the prognosis of patients; thus, PNLNR is a better predictor of prognosis.

Tumor size is a key factor affecting prognosis, and previous articles used a tumor diameter of $4 \mathrm{~cm}$ as the cutoff to analyze prognosis [18]. In this study, it was found that tumor size affected prognosis more than $T$ stage and pTNM stage, and patients with a tumor size larger than $4 \mathrm{~cm}$ also had a worse prognosis. Moreover, adjuvant chemotherapy is quite important for AEG patients who undergo resection. Oxaliplatin-based systemic chemotherapy has led to significant survival benefits. Elevated serum CEA and perineural invasion in pathology were also prognostic factors, which reminds us to conduct thorough preoperative evaluations, including for serum tumor biomarkers, and create detailed pathological reports.
As our study is a single-center retrospective study, we could not avoid some biases from incomplete patient data and heterogenous surgical operations. Because the information on the time of recurrence was incomplete, we did not conduct statistical analysis on the median time of recurrence and did not analyze the factors affecting recurrence. Our study was unable to record all complications in detail; therefore, we can only indirectly reflect on the influence of patient recovery on prognosis based on the length of hospital stay. A larger study, preferably a randomized controlled trial in multiple centers, is needed to standardize the surgical options for Siewert type II AEG patients.

\section{Conclusions}

Adjuvant chemotherapy, PNLNR, intraoperative blood transfusion, tumor size, perineural invasion, serum CEA, and duration of hospital stay after surgery are independent risk factors for survival in Siewert type II AEG patients who undergo resection. PNLNR is a better prognostic factor than pure $\mathrm{N}$ stage.

\section{Abbreviations}

AEG: Adenocarcinoma of the esophagogastric junction; PNLNR: Ratio of positive to negative lymph nodes; OS: Overall survival. 


\section{Acknowledgements}

The authors thank all the patients who participated in this study.

\section{Authors' contributions}

YDF designed the research, collected the data and wrote the paper; QXC designed the research and supervised the report; HYZ contributed to the statistical analysis; YHJ, QZ, JSL supervised the report. All authors read and approved the final manuscript.

\section{Funding}

No funding.

\section{Availability of data and materials}

The datasets used and/or analysed during the current study are available from the corresponding author on reasonable request.

\section{Ethics approval and consent to participate}

This study was reviewed and approved by the Research and Education Department of the Zhejiang Cancer Hospital. And consent was not required by ehics committee since its a retrospective study.

\section{Consent for publication}

Not applicable.

\section{Competing interests}

The authors declare that they have no competing interests.

\section{Author details}

${ }^{1}$ Department of Thoracic Surgery, Cancer Hospital of the University of Chinese Academy of Sciences(Zhejiang Cancer Hospital), Hangzhou 31000, China.

${ }^{2}$ Department of Cancer Biotherapy Center, First Affiliated Hospital, School of Medicine, Zhejiang University, Hangzhou 31000, China.

Received: 11 July 2020 Accepted: 22 October 2020

Published online: 30 November 2020

\section{References}

1. Zeng H, Zheng R, Zhang S, Zuo T, Xia C, Zou X, Chen W. Esophageal cancer statistics in China, 2011: Estimates based on 177 cancer registries. Thorac Cancer. 2016;7(2):232-7.

2. Liu S, Dai JY, Yao L, Li X, Reid B, Self S, Ma J, Chang Y, Feng S, Tapsoba Jde $D$, et al. Esophageal adenocarcinoma and its rare association with Barrett's Esophagus in Henan, China. PLoS ONE. 2014;9(10):e110348.

3. Liu K, Yang K, Zhang W, Chen X, Chen X, Zhang B, Chen Z, Chen J, Zhao $Y$, Zhou Z, et al. Changes of esophagogastric junctional adenocarcinoma and gastroesophageal reflux disease among surgical patients during 1988-2012: a single-institution high-volume experience in China. Ann Surg. 2016;263(1):88-95.

4. Hasegawa S, Yoshikawa T. Adenocarcinoma of the esophagogastric junction: incidence, characteristics, and treatment strategies. Gastric Cancer. 2010;13(2):63-73.

5. Trivers KF, Sabatino SA, Stewart SL. Trends in esophageal cancer incidence by histology, United States, 1998-2003. Int J Cancer. 2008;123(6):1422-8.

6. Dubecz A, Solymosi N, Stadlhuber RJ, Schweigert M, Stein HJ, Peters JH. Does the incidence of adenocarcinoma of the esophagus and gastric cardia continue to rise in the twenty-first century?-a SEER database analysis. J Gastrointest Surg. 2013;18(1):124-9.

7. Pohl H, Sirovich B, Welch HG. Esophageal adenocarcinoma incidence: are we reaching the peak? Cancer Epidemiol Biomarkers Prev. 2010;19(6):1468-70.

8. Carr JS, Zafar SF, Saba N, Khuri FR, El-Rayes BF. Risk factors for rising incidence of esophageal and gastric cardia adenocarcinoma. J Gastrointest Cancer. 2013;44(2):143-51.

9. Liu K, Zhang W, Chen X, Chen X, Yang K, Zhang B, Chen Z, Zhou Z, Hu J. Comparison on clinicopathological features and prognosis between esophagogastric junctional adenocarcinoma (siewert ii/iii types) and distal gastric adenocarcinoma: retrospective cohort study, a single institution, high volume experience in China. Medicine (Baltimore). 2015;94(34):e1386.

10. Rudiger Siewert J, Feith M, Werner M, Stein HJ. Adenocarcinoma of the esophagogastric junction: results of surgical therapy based on anatomi$\mathrm{cal} /$ topographic classification in 1,002 consecutive patients. Ann Surg. 2000:232(3):353-61.

11. Mine S, Kurokawa Y, Takeuchi H, Kishi K, Ito Y, Ohi M, Matsuda T, Hamakawa T, Hasegawa S, Yoshikawa T, et al. Distribution of involved abdominal lymph nodes is correlated with the distance from the esophagogastric junction to the distal end of the tumor in Siewert type II tumors. Eur J Surg Oncol. 2015;41(10):1348-53.

12. Wang WP, He SL, Yang YS, Chen LQ. Strategies of nodal staging of the TNM system for esophageal cancer. Ann Transl Med. 2018;6(4):77.

13. Sasako M, Sano T, Yamamoto S, Sairenji M, Arai K, Kinoshita T, Nashimoto A, Hiratsuka M. Japan Clinical Oncology G: Left thoracoabdominal approach versus abdominal-transhiatal approach for gastric cancer of the cardia or subcardia: a randomised controlled trial. Lancet Oncol. 2006;7(8):644-51.

14. Hulscher JB, van Sandick JW, de Boer AG, Wijnhoven BP, Tijssen JG, Fockens P, Stalmeier PF, ten Kate FJ, van Dekken H, Obertop H, et al. Extended transthoracic resection compared with limited transhiatal resection for adenocarcinoma of the esophagus. N Engl J Med. 2002;347(21):1662-9.

15. Omloo JM, Lagarde SM, Hulscher JB, Reitsma JB, Fockens P, van Dekken $H$ Ten-Kate FJ, Obertop H, Tilanus HW, van Lanschot JJ: Extended transthoracic resection compared with limited transhiatal resection for adenocarcinoma of the mid/distal esophagus: five-year survival of a randomized clinical trial. Ann Surg 2007, 246(6):992-1000 (discussion 1000-1001).

16. Houbiers JG, Brand A, van de Watering LM, Hermans J, Verwey PJ, Bijnen AB, Pahlplatz P, Eeftinck Schattenkerk M, Wobbes T, de Vries JE, et al. Randomised controlled trial comparing transfusion of leucocyte-depleted or buffy-coat-depleted blood in surgery for colorectal cancer. Lancet. 1994;344(8922):573-8.

17. Elwood NR, Martin AN, Turrentine FE, Jones RS, Zaydfudim VM. The negative effect of perioperative red blood cell transfusion on morbidity and mortality after major abdominal operations. Am J Surg. 2018;216(3):487-91.

18. Hoshino I, Gunji H, Ishige F, Iwatate $Y$, Takiguchi N, Ikeda A, Soda H, Tonooka T, Sato N, Kawahara K, et al. Surgical treatment strategy for esophagogastric junction cancers based on the tumor diameter. BMC Surg. 2019;19(1):152.

\section{Publisher's Note}

Springer Nature remains neutral with regard to jurisdictional claims in published maps and institutional affiliations. 